\title{
Definitive chemotherapy: a new frontier in the fight against laryngeal cancer
}

\author{
Missak Haigentz Jr $\cdot$ Carl E. Silver • \\ Alessandra Rinaldo · Alfio Ferlito
}

Received: 20 July 2009/Accepted: 31 August 2009/Published online: 22 September 2009

(C) Springer-Verlag 2009

\section{Introduction}

The hope of physicians during the nearly 60-year history of cancer chemotherapy has been to cure and to improve survival of patients with systemic therapy. While chemotherapy as a single treatment modality assists in symptom palliation or very modestly improves survival in some metastatic and incurable settings, the curative role of chemotherapy in most solid malignancies has been as an adjunct to or in conjunction with a definitive locoregional therapy, usually surgery or radiotherapy. The curative potential of chemotherapy alone has traditionally been limited to some hematological malignancies and testicular cancers. That concept is now changing.

Laryngeal cancer now joins the ranks of those select malignancies potentially curable with chemotherapy alone. This hypothesis, originally proposed and investigated by Laccourreye et al. [1] in a small series of patients, has been confirmed in recent publications [2-5].

\section{Haigentz Jr}

Division of Oncology, Department of Medicine,

Albert Einstein College of Medicine,

Montefiore Medical Center, Bronx, NY, USA

\section{E. Silver}

Departments of Surgery and Otolaryngology-Head and Neck Surgery, Albert Einstein College of Medicine, Montefiore Medical Center, Bronx, NY, USA

\author{
A. Rinaldo $\cdot$ A. Ferlito $(\square)$ \\ Department of Surgical Sciences, ENT Clinic, \\ University of Udine, Policlinico Universitario, \\ Piazzale S. Maria della Misericordia, 33100 Udine, Italy \\ e-mail: a.ferlito@uniud.it
}

\section{Reported studies}

In 1996, Laccourreye et al. [1] compared the results of 21 patients with T1-3 glottic squamous cell carcinoma who had complete clinical response (CCR) after cisplatin-fluorouracil chemotherapy and no further initial treatment with 37 comparable patients who received definitive radiotherapy or conservation surgery after a complete response to the same chemotherapy regimen. The local control rate was $70.7 \%$ in the group treated by chemotherapy alone and $97 \%$ in the group treated by radiation or surgery after CCR to chemotherapy $(p=0.002)$. Local recurrence in patients who had CCR managed with initial chemotherapy alone was always salvaged with partial laryngectomy or radiation therapy, which resulted in an overall $100 \%$ local control and laryngeal preservation rate within this group. While local recurrence affected nearly one-third of the patients treated by chemotherapy alone, the overall survival rate was actually higher (95.2\%) among the patients treated by chemotherapy alone than among the patients whose CCR was followed by conventional treatment with irradiation or surgery (possibly due to patient selection). The authors concluded that there is "clearly a significant subset of patients with chemocurable tumors who achieved both perfect preservation of structure-supporting voice and long-term survival after exclusive chemotherapy."

The same group [2] conducted a follow-up study, published in 2002, to analyze the consequences and management of local recurrence and to document the long-term results in patients with selected T1-3 glottic cancer who achieved CCR and were managed with platinum-based chemotherapy alone for cure. The cohort had grown to 35 patients with a minimum of 3 years follow-up, compared with 42 complete responders who received subsequent 
conventional treatment. Local control was achieved in $64.8 \%$ of patients, and salvage treatment in patients with local recurrence yielded $100 \%$ local control and laryngeal preservation rate. The 3- and 5-year actuarial survival estimates were 91.4 and $88.6 \%$, respectively. Overall, the causes of death were intercurrent disease in six patients and metachronous second primary tumor in four patients. Again, although local recurrence was noted in a third of patients with CCR, none of the patients who had local recurrence ultimately died from their disease or lost their larynx.

In 2007, Bonfils et al. [3] reported a retrospective study performed on 93 patients with laryngeal invasive squamous cell carcinoma managed with an induction chemotherapy protocol. Of these, 48 patients achieved a complete clinical, radiological, and histological response with a 3- and 5year actuarial survival rates for death by cancer of 95.6 and $79 \%$, respectively, and overall survival rates of 82.9 and $68.9 \%$, respectively. The authors concluded that chemotherapy alone had produced a definitive cure without detrimental effects on survival.

In 2009, Holsinger et al. [4] reported results of a prospective study at the M.D. Anderson Cancer Center, University of Texas, of 31 previously untreated patients with laryngeal cancer (T2-4, N0-1, M0), with tumors amenable to conservation laryngeal surgery. Patients received three to four cycles of paclitaxel, ifosfamide, and cisplatin (TIP) chemotherapy, and response was assessed histologically. Patients achieving pathologic complete response (pCR) received an additional three cycles of TIP and no other treatment. With TIP chemotherapy alone, 11 patients (37\%) achieved pCR, 10 of whom (33\%) remained alive at the time of the report, with durable disease remission and no evidence of recurrence over a median follow-up time of 5 years. The authors concluded that chemotherapy alone in selected patients with T2-4, N0-1 laryngeal cancer could provide durable disease remission at 5 years.

In the most recent report from the University of Paris, Holsinger et al. [5] reviewed all patients treated with chemotherapy alone from 1981 to 2004 resulting in a much larger cohort of 142 patients with a longer duration of follow-up than previously reported from that institution. No deaths were attributable to the chemotherapy treatment. The authors reported 5-year survival of $61.2 \%$, with metachronous second primaries and intercurrent disease being the main causes of death. Salvage therapy resulted in a $93 \%$ overall local control rate $(97.2 \%$ in patients with glottic cancer). Chemotherapy permitted successful modulation of local therapy in $54.9 \%$ of patients (78/142). Although this latter series [5] represents a retrospective review of a heterogeneous patient population, several important observations can be made. First, it is notable that the results of this study were achieved with platinum-based chemotherapy regimens that did not include taxanes or biological therapies; modern agents with proven clinical efficacy in treating systemic disease. Second, this study included patients with laryngeal as well as pharyngeal cancers. On multivariate analysis, however, the greatest benefits for exclusive chemotherapy were observed for patients with glottic tumors and with low comorbidity scores. Third, although few patients with nodal disease were included in the series, worse survival was noted for patients not initially staged with N0 disease. The authors concluded that, for selected patients, chemotherapy alone might provide long-term durable disease control. For patients with relapse after chemotherapy this approach does not diminish survival and maintains function in a majority of patients.

\section{Implications for the future}

While preliminary, with only one prospective study to date, the above research has demonstrated proof of concept for durable disease control with chemotherapy alone for selected patients with laryngeal cancer. Efforts at validating these results in future trials and expanding the study of exclusive chemotherapy in patients with nodal disease will be critical in developing a more widely applicable therapy. Future work should be directed to select markers of response to chemotherapy and thereby to identify which patients are optimally suited for this approach [5].

The local recurrence rate of approximately one-third of the patients treated by and responding to chemotherapy alone poses the greatest question of efficacy of this approach. Nevertheless, as documented by Holsinger et al. [4], failure appears limited to locoregional recurrences when close patient follow-up is employed, supporting the role of systemic therapy in treating and preventing distant micrometastatic disease. The main modification from present sequential neoadjuvant chemotherapy regimens would be a delay, possibly indefinitely, of the conventional definitive phase of treatment in patients who achieve complete histologic response from initial chemotherapy; substituting instead, several additional cycles of chemotherapy. Subsequent salvage treatment, administered when and if indicated, appears to produce the same rate of laryngeal preservation and overall survival as if the definitive treatment were given initially.

In the prospective study [4], 37\% (11/31) of patients achieved biopsy-confirmed complete response with chemotherapy alone; nearly all $(91 \%, 10 / 11)$ had no evidence of disease recurrence with a median follow-up time of 5 years. With a modern chemotherapy regimen, development of metachronous second primary cancers, a common 
cause of mortality of curatively treated head and neck cancer patients, also appears to be reduced. Given the potential value of this treatment, patient adherence with treatment and follow-up recommendations is critical to successful outcomes. This type of therapy is therefore clearly not advised for every patient with laryngeal cancer, and currently should be done only in the context of a clinical trial at centers with established multidisciplinary head and neck cancer programs where intensive follow-up with appropriate investigation is possible [6]. Validation of these results with controlled, multicenter clinical trials will be critical prior to widespread generalization of this approach [7].

Eighteen years have passed since the publication of the Veterans Affairs Laryngeal Cancer Study [8], and despite great advances in radiation-based curative nonsurgical therapies, patients and oncologists remain faced with decisions in balancing disease curability with management of acute and chronic toxicities and in a minority of patients, a risk of permanent impairment associated with curative radiotherapy. While cytotoxic chemotherapy can be associated with serious acute toxicity, no deaths were observed in these two recently published patient series $[4,5]$. However, the attractiveness of this approach centers on avoidance of an effective but occasionally morbid intervention. While laryngeal cancer remains an eminently curable disease with conventional treatment, it is commonly accepted medical knowledge that both surgery and irradiation are associated with permanent adverse effects on voice, deglutition, quality of life, and/or ability to treat subsequent new primary tumors. In a comprehensive review of the recent literature in laryngeal cancers, Silver et al. [9] have evaluated the known morbidity of non-surgical protocols as opposed to surgery followed by adjuvant radiotherapy. Both established treatments are associated with potential for long-term sequelae, including requirement for feeding tubes for two or more years and/or potential treatment-related death (e.g., pneumonia) within 3 years for patients treated with chemoradiotherapy and impaired voice function in patients treated with total laryngectomy. Reserving radiotherapy or surgery (either endoscopic laser surgery or open partial or total laryngectomy) for curative-intent treatment of recurrent or metachronous second primary disease may be meaningful to individuals wishing to preserve maximal laryngeal function with a definitive chemotherapy approach, though this has yet to be demonstrated. Bonfils et al. [3] indicated that salvage surgery is the best option in patients with local recurrence because resistance to chemotherapy may suggest resistance to radiotherapy. Further evaluation of patient quality of life outcomes and survivorship concerns will be important in future studies of chemotherapy as a single treatment modality.
These new investigations highlight a very important principle of clinical oncology: laryngeal cancer, as well as head and neck cancer in general, is a remarkably chemosensitive disease. The early studies of induction chemotherapy have demonstrated that previously untreated disease can frequently result in biopsy-confirmed complete responses, even in the setting of locoregionally advanced cancer, an observation exploited in current aggressive induction and sequential (induction followed by concurrent) chemoradiotherapy paradigms. Additionally, the observation that select laryngeal cancers can be curatively treated with chemotherapy alone provides a platform on which advances made in systemic therapies for patients with incurable disease can now be safely and directly translated to patients with potentially curable disease. Biological therapies found to improve survival in patients with unresectable recurrent or metastatic disease should certainly be incorporated in this setting. Furthermore, efforts to optimize therapy with novel chemotherapy paradigms should be considered. Addition of supradose intraarterial cisplatin chemotherapy, a regional treatment previously considered only for radiation sensitization, may perhaps further prevent regional recurrence and permit successful treatment of more advanced stage primary tumor and nodal disease. The role of high-dose chemotherapy with stem cell rescue, a treatment modality usually reserved for the few malignancies curable with chemotherapy alone, may also warrant investigation. Finally, demonstration of systemic chemotherapy as an effective single treatment modality in selected patients provides further hope for the development of effective and tolerable chemopreventive agents for head and neck cancers in at risk populations.

The potential availability of definitive chemotherapy will add one more curative treatment option for eligible patients, and another level of complexity for physicians seeking the optimal therapy recommendation for their patients. Identification and upfront selection of patients will be critical in individualizing therapy recommendations. In the VA Laryngeal Cancer Study, low T stage (T1-3 disease vs. T4 disease) was found to be the best predictor of response to induction chemotherapy [10]. Unfortunately, not much more is known about prediction of chemosensitivity in this disease. Tumor response following a few cycles of induction chemotherapy has been the traditional means of directing subsequent definitive management of patients with laryngeal cancer, and has been the basis for research on definitive chemotherapy to date. This paradigm inevitably results in the unnecessary treatment of a large number of patients who will not derive benefit from induction chemotherapy [7]. To optimally select patients, pre-treatment molecular predictors of response to chemotherapy must be identified and validated in the context of 
clinical trials, not only in studies of this approach, but also in induction and sequential chemoradiotherapy regimens where participating patients have also been previously untreated.

Physicians involved in the treatment of individuals with laryngeal cancer are now accustomed to novel treatment paradigms that challenge traditional oncological principles and result in improved organ preservation while preserving function and curative intent. The Veterans Affairs Laryngeal Cancer Study [8] and the RTOG 91-11 Intergroup study [11] have defined the evolving curative roles of induction chemotherapy and concurrent chemoradiotherapy, respectively, and have led to ongoing work with stateof-the-art chemoradiotherapy approaches. As a result of these landmark studies, laryngeal cancer has become the model for organ preserving and curative non-surgical therapies in clinical oncology [12]. Driven by both curative intent and improvement in the quality of life of patients with preserved larynx functionality, continued progress in curative chemotherapy for laryngeal cancer is expected. Access to specialized multidisciplinary treatment teams will remain the standard for high-quality patient care critical for successful clinical outcomes and meaningful clinical research in this field. As a result of current and future investigations, it is hoped that definitive chemotherapy will change the standard of care again for select patients with this disease and possibly serve as a model for curative chemotherapy in other solid tumors.

\section{References}

1. Laccourreye O, Brasnu D, Bassot V, Ménard M, Khayat D, Laccourreye H (1996) Cisplatin-fluorouracil exclusive chemotherapy for T1-T3N0 glottic squamous cell carcinoma complete clinical responders: five year results. J Clin Oncol 14:23312336

2. Laccourreye O, Veivers D, Bassot V, Ménard M, Brasnu D, Laccourreye H (2002) Analysis of local recurrence in patients with selected T1-3N0M0 squamous cell carcinoma of the true vocal cord managed with a platinum-based chemotherapy-alone regimen for cure. Ann Otol Rhinol Laryngol 111:315-321 (discussion 321-2)

3. Bonfils P, Trotoux J, Bassot V (2007) Chemotherapy alone in laryngeal squamous cell carcinoma. J Laryngol Otol 121:143148

4. Holsinger FC, Kies MS, Diaz EM Jr et al (2009) Durable longterm remission with chemotherapy alone for stage II to IV laryngeal cancer. J Clin Oncol 27:1976-1982

5. Holsinger FC, Lin HY, Bassot V, Laccourreye O (2009) Platinbased exclusive chemotherapy for selected patients with squamous cell carcinoma of the larynx and pharynx. Cancer 115:3909-3918

6. Forastiere AA, Shaha AR (2009) Chemotherapy alone for laryngeal preservation-is it possible? J Clin Oncol 27:1933-1934 (Editorial)

7. Hartl DM, Brasnu DF (2009) Chemotherapy alone for glottic carcinoma: a need for higher-level evidence. Ann Otol Rhinol Laryngol 118:543-545 (Editorial)

8. Wolf GT, Hong WK, Fisher SG et al (1991) Department of Veterans Affairs Laryngeal Cancer Study Group. Induction chemotherapy plus radiation compared with surgery plus radiation in patients with advanced laryngeal cancer. $\mathrm{N}$ Engl J Med 324: $1685-1690$

9. Silver CE, Beitler JJ, Shaha AR, Rinaldo A, Ferlito A (2009) Current trends in initial management of laryngeal cancer: the declining use of open surgery. Eur Arch Otolaryngol 266:13331352

10. Bradford CR, Wolf GT, Carey TE et al (1999) Predictive markers for response to chemotherapy, organ preservation, and survival in patients with advanced laryngeal carcinoma. Otolaryngol Head Neck Surg 121:534-538

11. Forastiere AA, Goepfert H, Maor M et al (2003) Concurrent chemotherapy and radiotherapy for organ preservation in advanced laryngeal cancer. N Engl J Med 349:2091-2098

12. Lefebvre J-L, Ang KK (2009) Larynx preservation clinical trial design: key issues and recommendations-a consensus. Head Neck 31:429-441 